\title{
A Rare Case Of Well Differentiated Papillary Mesothelioma Of Peritoneal Origin
}

\author{
Dr. B. Anil Kumar ${ }^{1}$, Professor, Dr. Sajana Gogineni ${ }^{2}$, Professor, \\ Dr. Channareddy Suneetha ${ }^{3}$, Assistant. Professor, Dr. Kalyan Chakravarthy ${ }^{4}$, \\ Professor, Dr. B. Nissy Jacintha ${ }^{5}$, PG \\ ${ }^{I}$ (Dept. of General Surgery, Dr. PSIMS \& RF, Chinoutpalli, AP, India) \\ 2,3,5 (Dept. of OBG, Dr. PSIMS \& RF, Chinoutpalli, AP, India) \\ ${ }^{4}$ (Dept. of Pathology, Dr. PSIMS \& RF, Chinoutpalli, AP, India
}

\begin{abstract}
Solitary well differentiated papillary mesothelioma is an unusual variant of epithelial mesothelioma. Most of them exhibit either benign or indolent behavior. Making the differential diagnosis between this rare tumor and serous papillary carcinoma can be problematic. We report here a case of a 24 year-old unmarried female with a well differentiated papillary mesothelioma of peritoneal origin.
\end{abstract}

Key Words: Mesothelioma; Ovary; well differentiated papillary mesothelioma.

\section{Introduction}

Well differentiated papillary mesothelioma (WDPM) is an unusual variant of epithelial mesothelioma. It occurs mainly in the peritoneum, and this tumor is most commonly seen in young women who have no history of asbestos exposure. It is often found incidentally at laparotomy that is done for other indications. ${ }^{1,2}$ WDPM rarely occurs at other sites, including the ovary, ${ }^{3,4}$ pericardium, ${ }^{5}$ and the tunica vaginalis ${ }^{6,7}$ The tumor is occasionally found on as a frozen sectioning because surgeons suspect this tumor to be other malignant tumors, such as diffuse malignant mesothelioma, or peritoneal dissemination from other tumor sites or ovarian serous papillary carcinoma. ${ }^{1}$ Most WDPMs exhibit either a benign or indolent behavior. ${ }^{1-8}$ Making the differential diagnosis between this rare tumor and serous papillary carcinoma can be problematic. WDPM must be distinguished from the significantly more aggressive lesions to avoid unnecessary treatment. ${ }^{1,8}$. We report here a case of a 24 year-old unmarried female with a well differentiated papillary mesothelioma of peritoneal origin.

\section{Case Report}

Miss X aged 24 years was bought to DR.PSIMS \& RF with pain of lower abdomen since 1 month duration. She has no h/o menstrual irregularities or history of asbestos exposure.

On examination her vitals are stable. P/A is soft. She is non co-operative on per rectal examination.

On gynecological US Rt ovary $-3.8 \times 1.8 \mathrm{~cm}$. A heterogenous lesion measuring $6.8 \times 3.9 \mathrm{~cm}$ seen in right adnexa in close approximation with anterosuperior border of right ovary peripheral and internal vascualrity noted within the lesion .

Her $\quad$ CA $125-472 \mathrm{u} / \mathrm{ml} ; \mathrm{CEA}-4.5 \mathrm{ng} / \mathrm{ml} ; \alpha \mathrm{FP}-2.1 \mathrm{IU} / \mathrm{ml} ; \mathrm{CA}-19.9-3.8 \mathrm{IU} / \mathrm{dl}$.

On CT scan right ovary- measures approximately $2.8 \times 1.9 \mathrm{~cm}$. Evidence of complex cystic lesion seen in Right adnexal region measuring approximately $6.8 \mathrm{~cm}$ in transverse diameter \& $4.6 \mathrm{~cm}$ anteroposteriorly. The lesions is in close contact with superolateral border of urinary bladder and superior to fundus of uterus. The lesion is in contact with right ovary . Lesion is showing nodularity in continuity with adjacent of cyst on post contrast study. At least two small cysts seen adjacent to the adnexal region on either side measuring $2 \mathrm{~cm}$ on each side possibly arising from respective ovarian tissue.

Laprotomy done - An irregular mass of size $5 \times 4 \mathrm{~cm}$ seen adjacent to the superior border of Right ovary from peritoneum seen. Mass is friable and easily removed. Grape like vesicles in appearance. mass removed \& sent for frozen section.

On frozen section -well differentiated papillary mesothelioma of peritoneal origin.

On histopathological examination - tumour showing papillary structures with columnar epithelial lining. The papillae have abundant stroma. Focal areas showing a complex papillary structures lined by cuboidal to columnar cells with a few cells showing eosinophilic and few cells have clear cytoplasm. Few cells exhibiting pleomerphium. At one focal area there is epithelial dipping into stroma forming tubules. There are necrotic foci with nuclear debris.

Patient has been referred to oncologist for further evaluation where it has been diagnosed as well differentiated papillary mesothelioma of peritoneal origin which has benign and indolent course.

The patient has remained well after a follow-up of seven months 


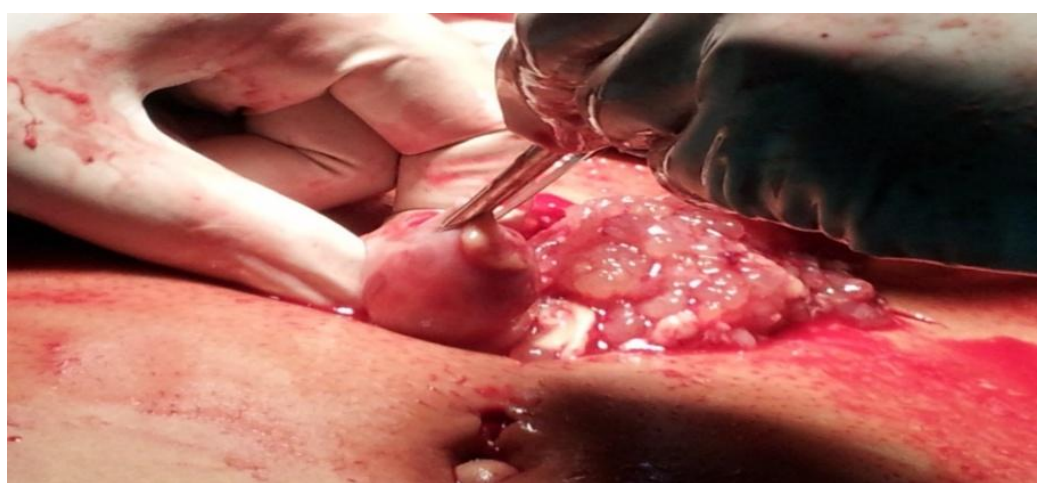

Pic 1: Irregular mass of size $5 \mathrm{x} 4 \mathrm{~cm}$ seen adjacent to the superior border of Right ovary from peritoneum seen.

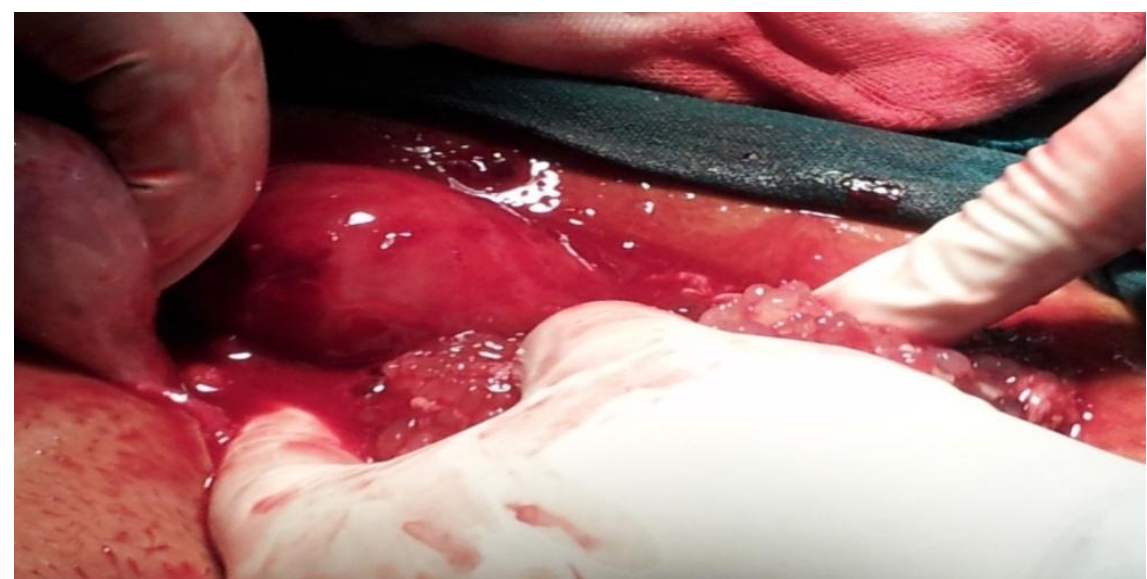

Pic 2: Grape like vesicle in appearance

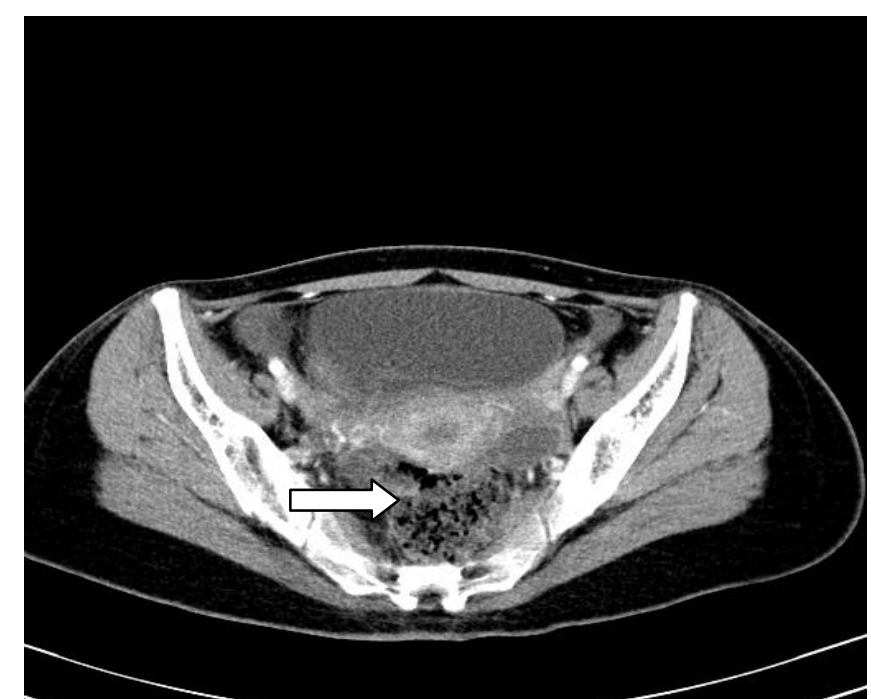

Pic 3: Evidence of complex cystic lesion ( 20 - 30 HU0 seen in Rt adnexal region measuring approximately 6. 8 $\mathrm{cm}$ in transverse diameter \& $4.6 \mathrm{~cm}$ anteroposteriorly 


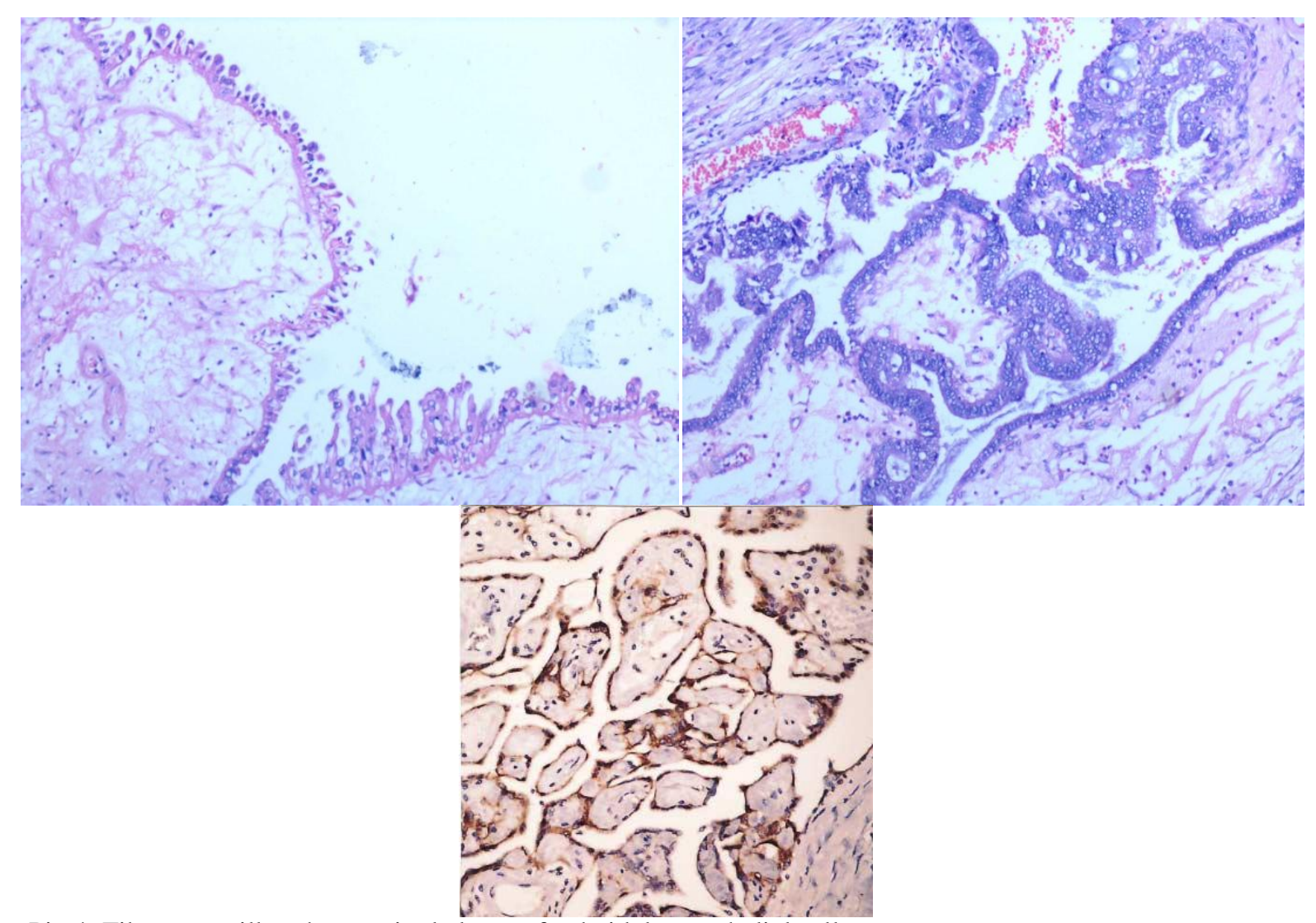

Pic 4: Fibrous papillae show a single layer of cuboidal mesothelial cells The tumor cells stain positively for calretinin (Immunohistochemical stain).

\section{Discussion}

WDPM is considered to be a benign subtype of mesothelioma that predominantly affects the peritoneum of women in the third and fourth decade. ${ }^{1,2}$ The histologic appearance of WDPM is that of thin fibrovascular papillae lined by relatively uniform cuboidal mesothelial cells. ${ }^{1}$ Psammoma bodies are occasionally encountered. ${ }^{1,9}$ WDPM must be differentiated from metastatic peritoneal adenocarcinoma, serous papillary adenocarcinoma, diffuse malignant mesothelioma and reactive mesothelial hyperplasia. ${ }^{1}$

The most important point in the differential diagnosis of this case was to rule out serous papillary adenocarcinoma of ovary. The absence of multilayering, cell stratification, cytologic atypia and mitotic activity supported a diagnosis of WDPM. ${ }^{1}$ Calretinin is a 29 - $\mathrm{kDa}$ calcium binding protein that is expressed in central and peripheral neural tissues, and in a limited number of nonneural tissues including normal and neoplastic mesothelium. ${ }^{10}$ Calretinin was previously identified to be the most sensitive $(88 \%)$ and specific $(100 \%)$ mesothelial marker to differentiate diffuse peritoneal mesothelioma in woman from serous papillary ovarian carcinoma and serous papillary peritoneal carcinoma. ${ }^{11}$ This case showed reactivity for calretinin and cytokeratin, but any reaction for CEA was negative. The immunoreactivity for CEA and CD15, and another glycoproteins, such as Ber-EP4, TAG-72, and MOC-31 is quite useful in supporting a diagnosis of adenocarcinoma. ${ }^{12}$

Diffuse malignant mesothelioma (DMM), in which well-differentiated papillary elements are prominent, can also be confused with WDPM. DMMs are usually bulkier than WDPMs and they demonstrate diffusely infiltrative cytologically atypical cells. ${ }^{9}$ Abnormal mitoses and bizarre cells are also found in DMM. ${ }^{13}$ .Patients with WDPM generally have a far better prognosis than those with DMM.

WDPM must also be distinguished from reactive mesothelial hyperplasia. Papillary structures may occur in mesothelial hyperplasia. ${ }^{14,15}$ However, in comparison the conspicuous papillary pattern of WDPM, such changes are rare in with hyperplasia. The lack of inflammation, the accompanying reactive changes in the adjacent serosal tissue and the relatively uniform or monotonous tumor cells are essential points for the diagnosis of WDPM. 


\section{Conclusion}

It is important to diagnose this tumor in order to refrain from an aggressive therapeutic approach. The histological findings that include the cytologic features and the immunohistochemical study results are important for making the correct diagnosis.

\section{References}

[1]. Daya D, McCaughey WT. Well-differentiated papillary mesothelioma of the peritoneum. A clinicopathologic study of 22 cases. Cancer 1990; 65: 292-6.

[2]. Goepel JR. Benign papillary mesothelioma of peritoneum: a histological, histochemical and ultrastructural study of six cases. Histopathology 1981; 5: 21-30.

[3]. Addis BJ, Fox H. Papillary mesothelioma of ovary. Histopathology 1983; 7: 287-98.

[4]. Alcantara Vazquez A, Oropeza Rechv G, Hernandez Gonzalez M, Rosales ML. Papillary mesothelioma of the ovarian surface. Presentation of a case. Ginecol Obstet Mex 1989; 57: 298-301.

[5]. Sane AC, Roggli VL. Curative resection of a well-differentiated papillary mesothelioma of the pericardium. Arch Pathol Lab Med 1995; 119: 266-7.

[6]. Chetty R. Well differentiated (benign) papillary mesothelioma of the tunica vaginalis. J Clin Pathol 1992: 45: 1029-30.

[7]. Xiao SY, Rizzo P, Carbone M. Benign papillary mesothelioma of the tunica vaginalis testis. Arch Pathol Lab Med 2000; 124: 1437.

[8]. McCaughey WT. Papillary peritoneal neoplasms in females. Pathol Annu 1985; 20: 387-404.

[9]. Daya D, McCaughey WT. Pathology of the peritoneum: a review of selected topics. Semin Diagn Pathol 1991; 8: 277-89.

[10]. Andressen C, Blumcke I, Celio MR. Calcium-binding proteins: selective markers of nerve cells. Cell Tissue Res 1993; 271: 181208.

[11]. Ordonez NG. Role of immunohistochemistry in distinguishing epithelial peritoneal mesotheliomas from peritoneal and ovarian serous carcinomas. Am J Surg Pathol 1998; 22: 1203-14.

[12]. Ordonez NG. The immunohistochemical diagnosis of epithelial mesothelioma. Hum Pathol 1999; 30: $313-23$.

[13]. Koss LG. Effusions in the presence of cancer. In Diagnostic Cytology and Its Histopathologic Bases. 4th ed. Philadelphia: JB Lippincott, 1992; 1116-84.

[14]. Foyle A, Al-Jabi M, McCaughey WT. Papillary peritoneal tumors in women. Am J Surg Pathol 1981; 5: 241-9.

[15]. McCaughey WTE, Al-Jabi M. Differentiation of serosal hyperplasia and neoplasia in biopsies. Pathol Annu 1986; 21 : 271-93. 